\title{
ON THE SECOND GAUSSIAN CURVATURE OF RULED SURFACES IN EUCLIDEAN 3-SPACE
}

\author{
DAE WON YOON
}

\begin{abstract}
In this paper, we mainly investigate non-developable ruled surface in a 3-dimensional Euclidean space satisfying the equation $K_{I I}=K H$ along each ruling, where $K$ is the Gaussian curvature, $H$ is the mean curvature and $K_{I I}$ is the second Gaussian curvature.
\end{abstract}

\section{Introduction}

The inner geometry of the second fundamental form has been a popular research topic for ages. It is readily seen that the second fundamental form of a surface is nondegenerate if and only if a surface is non-developable.

On a non-developable surface $M$, we can consider the Gaussian curvature $K_{I I}$ of the second fundamental form which is regarded as a new Riemannian metric. The curvature $K_{I I}$ will be called the second Gaussian curvature of the surface $M$ (cf. [2]).

For the study of the second Gaussian curvature, D. Koutroufiotis ([9]) has shown that a closed ovaloid is a sphere if $K_{I I}=c K$ for some constant $c$ or if $K_{I I}=\sqrt{K}$, where $K$ is the Gaussian curvature. Th. Koufogiorgos and T. Hasanis ([8]) proved that the sphere is the only closed ovaloid satisfying $K_{I I}=H$, where $H$ is the mean curvature. Also, W. Kühnel ([10]) studied surfaces of revolution satisfying $K_{I I}=H$. One of the natural generalizations of surfaces of revolution is the helicoidal surfaces. In [1] C. Baikoussis and Th. Koufogiorgos proved that the helicoidal surfaces satisfying $K_{I I}=H$ are locally characterized by constancy of the ratio of the principal curvatures. On the other hand, D. E. Blair and Th. Koufogiorgos ([2]) investigated a non-developable ruled surface in a 3-dimensional Euclidean space $\mathbb{E}^{3}$ satisfying the condition

$$
a K_{I I}+b H=\text { constant }, \quad 2 a+b \neq 0,
$$

along each ruling. Also, they proved that a ruled surface with vanishing second Gaussian curvature is a helicoid.

Received January 21, 2005.

2000 Mathematics Subject Classification. 53A05, 53A10, 53B25.

Key words and phrases. Helicoid, minimal surface, ruled surface, Second Gaussian curvature, Mean curvature, Gaussian curvature.

This work was supported by Korea Research Foundation Grant(KRF-2004-041-C00039). 
Recently, the present author ([16]) studied a non-developable ruled surface in a 3dimensional Euclidean space $\mathbb{E}^{3}$ satisfying the conditions

$$
\begin{aligned}
a H+b K & =\text { constant }, \quad a \neq 0, \\
a K_{I I}+b K & =\text { constant }, \quad a \neq 0,
\end{aligned}
$$

along each ruling.

In particular, if it satisfies the condition (1.2), then a surface is called a linear Weingarten surface (see [11]).

On the other hand, in [7] Y. H. Kim and the present author investigated a nondevelopable ruled surface in a 3-dimensional Lorentz-Minkowski space $\mathbb{L}^{3}$ satisfying the conditions (1.1), (1.2) and (1.3). In [13] W. Sodsiri studied a non-developable ruled surface in $\mathbb{L}^{3}$ with non-null rulings such that the linear combination $a K_{I I}+b H+c K$ is constant along ruling.

In this article, we investigate a non-developable ruled surface in a Euclidean 3 -space $\mathbb{E}^{3}$ satisfying the condition

$$
K_{I I}=K H
$$

along each ruling.

On the other hand, many geometers have been interested in studying submanifolds of Euclidean and pseudo-Euclidean space in terms of the so-called finite type immersion ([3]). Also, such a notion can be extended to smooth maps on submanifolds, namely the Gauss map ([4]). The Gauss map $G$ on a submanifold $M$ of a Euclidean space or pseudo-Euclidean space is said to be of pointwise 1-type if $\Delta G=f G$ for some smooth function $f$ on $M$ where $\Delta$ denotes the Laplace operator defined on $M([6])$.

In [5] M. Choi and Y. H. Kim proved the following theorem which will be useful to prove our theorems in this paper.

Theorem 1.1.([5]) Let $M$ be a non-cylindrical ruled surface in a 3-dimensional Euclidean space. Then, the Gauss map is of pointwise 1-type if and only if $M$ is an open part of a helicoid.

\section{Preliminaries}

Let $\mathbb{E}^{3}$ be a 3 -dimensional Euclidean space with the metric $<,>=d x_{1}^{2}+d x_{2}^{2}+d x_{3}^{2}$, where $\left(x_{1}, x_{2}, x_{3}\right)$ is a standard rectangular coordinate system of $\mathbb{E}^{3}$.

We denote a surface $M$ in $\mathbb{E}^{3}$ by

$$
x(s, t)=\left\{x_{1}(s, t), x_{2}(s, t), x_{3}(s, t)\right\} .
$$

Then the first fundamental form $I$ of the surface $M$ is defined by

$$
\begin{gathered}
I=E d s^{2}+2 F d s d t+G d t^{2}, \\
E=<x_{s}, x_{s}>, \quad F=<x_{s}, x_{t}>, \quad G=<x_{t}, x_{t}>, \quad x_{s}=\frac{\partial x(s, t)}{\partial s} .
\end{gathered}
$$


We define the second fundamental form $I I$ of $M$ by

$$
\begin{aligned}
I I & =e d s^{2}+2 f d s d t+g d t^{2}, \\
e & =\frac{1}{\sqrt{E G-F^{2}}} \operatorname{det}\left(x_{s} x_{t} x_{s s}\right), \\
f & =\frac{1}{\sqrt{E G-F^{2}}} \operatorname{det}\left(x_{s} x_{t} x_{s t}\right), \\
g & =\frac{1}{\sqrt{E G-F^{2}}} \operatorname{det}\left(x_{s} x_{t} x_{t t}\right) .
\end{aligned}
$$

Using classical notation above, the Gaussian curvature $K$ is defined by (See, [14, p. 112])

$$
K=\frac{1}{\left(E G-F^{2}\right)^{2}}\left\{\left|\begin{array}{ccc}
-\frac{1}{2} E_{t t}+F_{s t}-\frac{1}{2} G_{s s} & \frac{1}{2} E_{s} & F_{s}-\frac{1}{2} E_{t} \\
F_{t}-\frac{1}{2} G_{s} & E & F \\
\frac{1}{2} G_{t} & F & G
\end{array}\right|-\left|\begin{array}{ccc}
0 & \frac{1}{2} E_{t} & \frac{1}{2} G_{s} \\
\frac{1}{2} E_{t} & E & F \\
\frac{1}{2} G_{s} & F & G
\end{array}\right|\right\},
$$

or equivalently,

$$
K=\frac{e g-f^{2}}{E G-F^{2}}
$$

On the other hand, the mean curvature $H$ is given by

$$
H=\frac{1}{2} \frac{E g-2 F f+G e}{E G-F^{2}} .
$$

At this stage we are able to compute the second Gaussian curvature $K_{I I}$ of a nondevelopable surface in $\mathbb{E}^{3}$ by replacing $E, F, G$ by the components of the second fundamental form $e, f, g$ respectively in (2.1). Thus, the second Gaussian curvature $K_{I I}$ is given by

$$
K_{I I}=\frac{1}{\left(e g-f^{2}\right)^{2}}\left\{\begin{array}{ccc}
-\frac{1}{2} e_{t t}+f_{s t}-\frac{1}{2} g_{s s} & \frac{1}{2} e_{s} & f_{s}-\frac{1}{2} e_{t} \\
f_{t}-\frac{1}{2} g_{s} & e & f \\
\frac{1}{2} g_{t} & f & g
\end{array}|-| \begin{array}{ccc}
0 & \frac{1}{2} e_{t} & \frac{1}{2} g_{s} \\
\frac{1}{2} e_{t} & e & f \\
\frac{1}{2} g_{s} & f & g
\end{array} \mid\right\}
$$

It is well known that a minimal surface has vanishing second Gaussian curvature but that a surface with vanishing second Gaussian curvature need not be minimal.

\section{Main Results}

In this section we classify a non-developable ruled surface in a Euclidean 3-space $\mathbb{E}^{3}$ satisfying the equations (1.4). It is well known that a cylindrical ruled surface is developable, i.e., the Gaussian curvature $K$ is identically zero. Therefore, the second fundamental form $I I$ is degenerate. Thus, non-cylindrical ruled surfaces are meaningful for our study. 
Theorem 3.1. A non-developable ruled surface in a Euclidean 3 -space $\mathbb{E}^{3}$ satisfying the condition $K_{I I}=K H$ along each ruling is a piece of a helicoid.

Proof. Let $M$ be a non-developable ruled surface in $\mathbb{E}^{3}$. Then the parametrization for $M$ is given by

$$
x=x(s, t)=\alpha(s)+t \beta(s)
$$

such that $\left.\langle\beta, \beta\rangle=1,<\beta^{\prime}, \beta^{\prime}\right\rangle=1$ and $\left\langle\alpha^{\prime}, \beta^{\prime}\right\rangle=0$. In this case $\alpha$ is the striction curve of $x$, and the parameter is the arc-length on the spherical curve $\beta$. And we have the natural frame $\left\{x_{s}, x_{t}\right\}$ given by $x_{s}=\alpha^{\prime}+t \beta^{\prime}$ and $x_{t}=\beta$. Then, the first fundamental form of the surface is given by $E=<\alpha^{\prime}, \alpha^{\prime}>+t^{2}, F=<\alpha^{\prime}, \beta>$ and $G=1$. For later use, we define the smooth functions $Q, J$ and $D$ as follows :

$$
Q=<\alpha^{\prime}, \beta \times \beta^{\prime}>\neq 0, \quad J=<\beta^{\prime \prime}, \beta^{\prime} \times \beta>, \quad D=\sqrt{E G-F^{2}} .
$$

In terms of the orthonormal basis $\left\{\beta, \beta^{\prime}, \beta \times \beta^{\prime}\right\}$ we obtain

$$
\alpha^{\prime}=F \beta+Q \beta \times \beta^{\prime}, \quad \beta^{\prime \prime}=-\beta-J \beta \times \beta^{\prime}, \quad \alpha^{\prime} \times \beta=Q \beta^{\prime},
$$

which imply $E G-F^{2}=Q^{2}+t^{2}$ and the unit normal vector $N$ is given by

$$
N=\frac{1}{D}\left(\alpha^{\prime} \times \beta+t \beta^{\prime} \times \beta\right)=\frac{1}{D}\left(Q \beta^{\prime}-t \beta \times \beta^{\prime}\right) .
$$

Therefore, the components $e, f$ and $g$ of the second fundamental form are expressed as

$$
e=\frac{1}{D}\left(Q(F+Q J)-Q^{\prime} t+J t^{2}\right), \quad f=\frac{Q}{D} \neq 0, \quad g=0 .
$$

Thus, using the dates described above and (2.2), we obtain

$$
\begin{aligned}
K_{I I} & =\frac{1}{f^{4}}\left(f f_{t}\left(f_{s}-\frac{1}{2} e_{t}\right)-f^{2}\left(-\frac{1}{2} e_{t t}+f_{s t}\right)\right) \\
& =\frac{1}{2 Q^{2} D^{3}}\left(J t^{4}+Q(F+2 Q J) t^{2}-2 Q^{2} Q^{\prime} t+Q^{3}(Q J-F)\right) .
\end{aligned}
$$

Furthermore, the mean curvature $H$ and the Gaussian curvature $K$ are given respectively by

and

$$
H=\frac{1}{2 D^{3}}\left(J t^{2}-Q^{\prime} t+Q(Q J-F)\right)
$$

$$
K=-\frac{Q^{2}}{D^{4}}
$$

We now differentiate $K_{I I}, H$ and $K$ with respect to $t$, the results are

$$
\begin{aligned}
\left(K_{I I}\right)_{t} & =\frac{1}{2 Q^{2} D^{5}}\left(J t^{5}+Q(2 Q J-F) t^{3}+4 Q^{2} Q^{\prime} t^{2}+Q^{3}(5 F+Q J) t-2 Q^{4} Q^{\prime}\right) \\
H_{t} & =\frac{1}{2 D^{5}}\left(-J t^{3}+2 Q^{\prime} t^{2}+Q(-Q J+3 F) t-Q^{2} Q^{\prime}\right)
\end{aligned}
$$

and 


$$
K_{t}=\frac{4 Q^{2}}{D^{6}} t
$$

Suppose that the surface satisfies the condition (1.4). Then, by (3.1)-(3.6) we can show that the coefficients of the power of $t$ are as follows:

$$
\begin{aligned}
t^{9}: & J=0, \\
t^{7}: & 4 Q^{2} J-Q F=0, \\
t^{6}: & 4 Q^{2} Q^{\prime}=0, \\
t^{5}: & 6 Q^{4} J+3 Q^{3} F=0, \\
t^{4}: & 6 Q^{4} Q^{\prime}=0, \\
t^{3}: & 4 Q^{6} J+9 Q^{5} F-5 Q^{4} J=0, \\
t^{2}: & 6 Q^{4} Q^{\prime}=0, \\
t^{1}: & 5 Q^{7} F+Q^{8} J+7 Q^{5} F-5 Q^{6} J=0, \\
t^{0}: & Q^{8} Q^{\prime}+Q^{6} Q^{\prime}=0,
\end{aligned}
$$

which imply $J=F=Q^{\prime}=0$. Thus, from (3.1) and (3.2) the second Gaussian curvature and the mean curvature are identically zero. Consequently, the surface is locally a helicoid. This completes the proof.

Combining the results of Theorem 1.1, our Theorem 3.1 and main Theorems in [16], we have

Theorem 3.2. Let $M$ be a non-developable ruled surface in a Euclidean 3-space. Then, the following are equivalent :

1. $M$ is piece of a helicoid.

2. $M$ has pointwise 1-type Gauss map.

3. $M$ satisfies the equation $a K_{I I}+b K+c=0, a \neq 0, b, c \in \mathbb{R}$ along each ruling.

4. $M$ satisfies the equation $a H+b H+c=0, a \neq 0, b, c \in \mathbb{R}$ along each ruling.

5. $M$ satisfies the equation $K_{I I}=K H$ along each ruling.

\section{References}

[1] C. Baikoussis and Th. Koufogiorgos, On the inner curvature of the second fundamental form of helicoidal surfaces, Arch. Math. 68(1997), 169-176.

[2] D. E. Blair and Th. Koufogiorgos, Ruled surfaces with vanishing second Gaussian curvature, Mh. Math. 113(1992), 177-181.

[3] B.-Y. Chen, Total Mean Curvature and Submanifolds of Finite Type, World Scientific Publ., 1984.

[4] B.-Y. Chen and P. Piccinni, Submanifolds with finite type Gauss map, Bull. Austral. Math. Soc. 35(1987), 161-186.

[5] M. Choi and Y. H. Kim, Characterization of the helicoid as ruled surfaces with pointwise 1-type Gauss map, Bull. Korean Math. Soc. 38(2001), 753-761.

[6] Y. H. Kim and D. W. Yoon, Ruled surfaces with pointwise 1-type Gauss map, J. Geometry and Physics 34(2000), 191-205.

[7] Y. H. Kim and D. W. Yoon, Characterization of ruled surfaces in Minkowski 3-spaces, J. Geometry and Physics 49(2004), 89-100. 
[8] Th. Koufogiorgos and T. Hasanis, A characteristic property of the sphere, Proc. Amer. Math. Soc. 67(1977), 303-305.

[9] D. Koutroufiotis, Two characteristic properties of the sphere, Proc. Amer. Math. Soc. 44(1974), 176-178.

[10] W. Kühnel, Zur inneren Krümmung der zweiten Grundform, Monatsh. Math. 91(1981), 241-251.

[11] H. Rosenberg and R. Sa Earp, The geometry of properly embedded special surfaces in $\mathbb{R}^{3}$; e.g., surfaces satisfying $a H+b K=1$, where $a$ and $b$ are positive, Duke Math. J. 73(1994), 291-306.

[12] R. Schneider, Closed convex hypersurfaces with second fundamental form of constant curvature, Proc. Amer. Math. Soc. 35(1972), 230-233.

[13] W. Sodsiri, Ruled linear Weingarten surface in Minkowski 3-space, Soochow J. Math. 29(2003), 435-443.

[14] D. J. Struik, Differential Geometry, Reading, Addison-Wesley, MA, 1961.

[15] I. V. de Woestijne, Minimal surfaces in the 3-dimensional Minkowski space Geomety and Topology of submanifolds, II, World Scientific, Singapore, 1990, 344-369.

[16] D. W. Yoon, Some properties of the helicoid as ruled surfaces, JP Jour. Geometry and Topology (2002), 141-147.

Department of Mathematics Education and RINS, Gyeongsang National University, Jinju 660701, South Korea.

E-mail: dwyoon@gsnu.ac.kr 